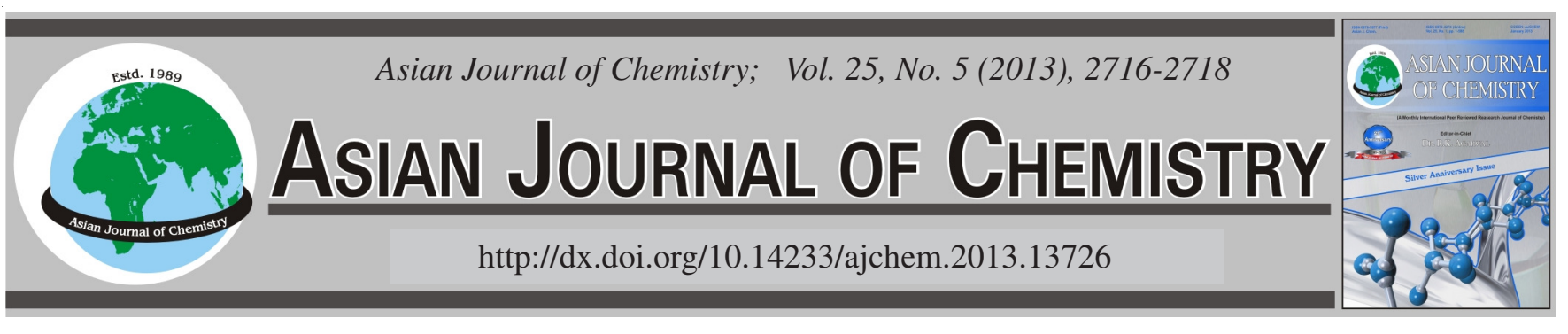

\title{
Synthesis and Spectral Studies of Some \\ 2-Oxopyrimidine-1(2H)-yl]-pyrrolidine-2,5-dione Derivatives
}

\author{
ZÜLBIYE ÖNAL
}

Department of Chemistry, Erciyes University, Kayseri, Turkey

Corresponding author: Fax: +90 352 4374931; Tel: +90 352 2076666, E-mail: zulbiye@erciyes.edu.tr

1-Amino-5-(4-methylbenzoyl)-4-(4-methylphenyl)pyrimidine-2(1H)-one/-thione (1a,b) reacted with various carboxylic anhydrides (2a-d) under different conditions and given the new amide and imide derivatives $(\mathbf{3 a}-\mathbf{h})$. The newly synthesized compounds were characterized by elemental analysis, IR, ${ }^{1} \mathrm{H}$ and ${ }^{13} \mathrm{C}$ NMR spectral data. All the compounds were compared with their previous analogues.

Key Words: $N$-Aminopyrimidine derivatives, Anhydride, Condensation, Amide, Imide.

\section{INTRODUCTION}

Pyrimidines are interested in biological and medicinal properties (herbicidal, antibacterial, antifungal, antiviral ${ }^{1-4}$. Some of them are frequently encountered in many drugs used for the treatment of hypothyroidy, hypertension, cancer chemotherapy or HIV infection ${ }^{5-8}$. 1-Amino-5-(4-methylbenzoyl)4-(4-methylphenyl)pyrimidine-2(1H)-one/-thione are synthesized in two steps from 4-(4-methylbenzoyl)-5-(4-methylphenyl)furan-2,3-dione ${ }^{9,10}$. It should start with a nucleophilic attack of the $-\mathrm{NH}_{2}$ group of semi-/thiosemicarbazones at the C-5 position of the furandione ring similar to a Micheal-type addition ${ }^{10}$. Their hydrolysis afforded the 1-aminopyrimidine derivatives exhibiting a free $-\mathrm{NH}_{2}$ moiety, which were applied to several subsequent reactions. The reactions of 1-aminopyrimidine derivatives with 1,3-dicarbonyl compounds, isocyanates and isothiocyanates have been reported in different conditions $^{11-17}$.

For these reasons, the aim of this study was to synthesized various pyrimidine derivatives to make notable contributions to this class of heterocyclic compounds that are generally well known for their potential biologicial activities ${ }^{1-8}$. In the present study, we carried out the reactions between 1-amino-5-(4methylbenzoyl)-4-(4-methylphenyl)pyrimidine-2(1H)-one/thione (1a,b) with succinic anhydride phthalic anhydride, benzoic anhydride and maleic anhydride (2a-d), respectively as shown in Scheme-I.

\section{EXPERIMENTAL}

Melting points were determined on an electrothermal 9200 apparatus and are uncorrected. Microanalyses were performed on a Leco-932 CHNS-O elemental analyser, model 1108. A Shimadzu FT-IR-8400 model spectrophotometer was used for IR spectra (4000-400 $\mathrm{cm}^{-1}$ region), using ATR techniques. The ${ }^{1} \mathrm{H}$ - and ${ }^{13} \mathrm{C}$-NMR spectra were measured with a Bruker Avance III $400 \mathrm{MHz}$ spectrometer and the chemical shifts were recorded in ppm units. After completion of the reactions, solvents were evaporated with a rotary evaporator (Buchi RE model 111). The reactions were followed by TLC using DC Alufolien Kieselgel 60 F254 Merck and a Camag TLC lamp $(254 / 366 \mathrm{~nm})$. Solvents and all other chemical reagents were purchased from commercial suppliers and were of reagent grade quality. Solvents were dried by refluxing with the appropriate drying agents and distilled before use.

$N$-[5-(4-methylbenzoyl)-4-(4-methylphenyl)-2oxopyrimidine-1(2H)-yl]pyrrolidine-2,5-dione (3a): $0.2 \mathrm{~g}$ of 1-Amino-5-(4-methylbenzoyl)-4-(4-methylphenyl)pyrimidine-2(1H)-one (1a) and $0.62 \mathrm{~g}$ succinic anhydride (2a) (1:10 molar ratio) were heated at $180{ }^{\circ} \mathrm{C}$ for at $1 \mathrm{~h}$ without any solvent. Then, the residue was treated with dry ether and filtered and the so formed crude product was recrystallized from $n$-butanol; yield $0.15 \mathrm{~g}(60 \%)$, white crystals; m.p. 330 ${ }^{\circ} \mathrm{C}$; IR: v = 3030 (aromatic C-H), 2930 (aliphatic C-H), 17401680-1650 $(\mathrm{C}=\mathrm{O}), 1580-1500(\mathrm{C}=\mathrm{C}$ and $\mathrm{C}=\mathrm{N}), 1220-1050$ (anhydride's C-O stretch), 800-700 $\mathrm{cm}^{-1}$ (pyrimidine ring); ${ }^{1} \mathrm{H}$ NMR (DMSO- $d_{6}$ ): $\delta=8.90$ (s, 1H, pyrimidine ring.), 7.776.84 (m, 8H, ArH), 2.60-2.45 ppm (- $\left.\mathrm{CH}_{2}-\mathrm{CH}_{2}-\right), 2.25,2.05$ ppm (s, 6H, 2x - $\left.\mathrm{CH}_{3}\right) ;{ }^{13} \mathrm{C}$ NMR (DMSO- $\left.d_{6}\right): \delta=191.50(\mathrm{~s}$, benzoyl's $\mathrm{C}=\mathrm{O}$ ), 162.30 (s, anhydride's $2(\mathrm{C}=\mathrm{O}), 152.30(\mathrm{~s}$, pyrimidine ring, $\mathrm{C}=\mathrm{O}), 143.01-122.20$ ( $\mathrm{m}$, aromatic $\mathrm{C}), 31.15$ (s, $\left.-\mathrm{CH}_{2}-\mathrm{CH}_{2}-\right), 23.15,21.14$ ppm (s, $2 \times \mathrm{CH}_{3} \mathrm{C}_{6} \mathrm{H}_{4}-$ ). Elemental 
analysis (\%): Found (calcd.): $\mathrm{C}=68.55$ (68.82), $\mathrm{H}=4.80$ (4.77), $\mathrm{N}=10.51$ (10.47).

$\mathrm{N}$-[5-(4-methylbenzoyl)-4-(4-methylphenyl)-2oxopyrimidine-1(2H)-yl]pyrrolidine-2,5-dione (3b): $0.2 \mathrm{~g}$ of 1-Amino-5-(4-methylbenzoyl)-4-(4-methylphenyl)pyrimidine-2(1H)-thione (1b) and $0.59 \mathrm{~g}$ succinic anhydride (2a) (1: 10 molar ratio) were heated at $170^{\circ} \mathrm{C}$ for at $2 \mathrm{~h}$ without any solvent. Then, the residue was treated with dry ether and filtered and the so formed crude product was recrystallized from $n$-butanol; yield $0.16 \mathrm{~g}$ (66.6\%), yellow crystals; m.p. $330{ }^{\circ} \mathrm{C}$; IR: $v=3040$ (aromatic C-H), 2945 (aliphatic C-H), 1745-1680-1650 (C=O), 1590-1510 ( $=\mathrm{C}$ and $\mathrm{C}=\mathrm{N}), 1240$ $\left(\mathrm{C}=\mathrm{S}\right.$ ), 1220-1050 (anhydride's C-O stretch), 800-700 $\mathrm{cm}^{-1}$ (pyrimidine ring); ${ }^{1} \mathrm{H}$ NMR (DMSO- $\left.d_{6}\right): \delta=8.80(\mathrm{~s}, 1 \mathrm{H}$, pyrimidine ring), 7.80-6.70 (m, $8 \mathrm{H}, \mathrm{ArH}), 2.50-2.45 \mathrm{ppm}$ $\left(-\mathrm{CH}_{2}-\mathrm{CH}_{2}-\right), 2.25,2.15 \mathrm{ppm}\left(\mathrm{s}, 6 \mathrm{H}, 2 \mathrm{x}-\mathrm{CH}_{3}\right) ;{ }^{13} \mathrm{C} \mathrm{NMR}$ (DMSO- $d_{6}$ ): $\delta=190.10$ (s, benzoyl's $\mathrm{C}=\mathrm{O}$ ), 171.30 (s, pyrimidine ring, $\mathrm{C}=\mathrm{S}), 162.30$ (s, anhydride's $2(\mathrm{C}=\mathrm{O}), 145.01$ 115.20 (m, aromatic C), $31.15\left(-\mathrm{CH}_{2}-\mathrm{CH}_{2}-\right), 23.15,21.14$ ppm (s, $\left.2 \mathrm{xCH}_{3} \mathrm{C}_{6} \mathrm{H}_{4}-\right)$. Elemental analysis (\%): Found (calcd.): $\mathrm{C}=66.45$ (66.17), $\mathrm{H}=4.31$ (4.59), $\mathrm{N}=10.23$ (10.07), $\mathrm{S}=$ 8.05 (7.48).

2-[5-(4-Methylbenzoyl)-4-(4-methylphenyl)-2-oxopyrimidine-1(2H)-yl]-1H-isoindole-1,3(2H)-dione (3c): $0.2 \mathrm{~g}$ of 1-Amino-5-(4-methylbenzoyl)-4-(4-methylphenyl)pyrimidine2(1H)-one (1a), a large excess of phthalic anhydride (2b), 0.88 $\mathrm{g}$ (molar ratio 1: 10) were homogeneously mixed. The mixture in a $50 \mathrm{~mL}$ round bottomed flask by fitting calcium chloride guard tube was heated at $190{ }^{\circ} \mathrm{C}$ for $1 \mathrm{~h}$ without any solvent. After cooling to room temperature the residue was treated with dry ether and the so formed crude product recrystallized from acetic acid; yield: $0.15 \mathrm{~g}(57 \%)$, white crystals; m.p. $305^{\circ} \mathrm{C}$; IR: $v=3500-3400$ (carbonyl overtone, $\mathrm{C}=\mathrm{O}$ ), 3040 (aromatic C-H), 2950 (aliphatic C-H), 1745-1670-1640 (C=O), 15401480 (aromatic ring, skeleton vib.), 1220-1050 (anhydride $\mathrm{C}-\mathrm{O}$ stretch), 800-700 (pyrimidine ring); ${ }^{1} \mathrm{H}$ NMR (DMSO$\left.d_{6}\right): \delta=8.90 \mathrm{ppm}(\mathrm{s}, 1 \mathrm{H}$, pyrimidine ring), 7.90-6.79 (m, $15 \mathrm{H}$, aromatic), 2.30-2.15 ppm (s, 6H, 2x- $\left.\mathrm{CH}_{3}\right) ;{ }^{13} \mathrm{C} \mathrm{NMR}$ (DMSO- $d_{6}$ ): $\delta=198.51$ (s, benzoyl's $\mathrm{C}=\mathrm{O}$ ), 160.10 (s, anhydride's $2(\mathrm{C}=\mathrm{O}), 151.30$ (s, pyrimidine ring, $\mathrm{C}=\mathrm{O}$ ), 145.01115.20 (m, aromatic C), 23.15, 21.14 ppm (s, $2 \mathrm{xCH}_{3} \mathrm{C}_{6} \mathrm{H}_{4}-$ ). Elemental analysis (\%): Found (calcd.): $\mathrm{C}=72.32$ (72.15), $\mathrm{H}$ $=4.01$ (4.26), $\mathrm{N}=9.18$ (9.35).

2-[5-(4-Methylbenzoyl)-4-(4-methylphenyl)-2-thioxopyrimidine-1(2H)-yl]-1H-isoindole-1,3(2H)-dione(3d): 0.2 $\mathrm{g}$ of 1-Amino-5-(4-methylbenzoyl)-4-(4-methylphenyl)pyrimidine-2(1H)-thione (1b), a large excess of phthalic anhydride (2b), $0.88 \mathrm{~g}$ (molar ratio 1: 10) were homogeneously mixed. The mixture in a $50 \mathrm{~mL}$ round bottomed flask by fitting calcium chloride guard tube was heated at $190{ }^{\circ} \mathrm{C}$ for $2 \mathrm{~h}$ without any solvent. After cooling to room temperature the residue was treated with dry ether and the so formed crude product recrystallized from acetic acid; yield: $0.17 \mathrm{~g}(61 \%)$, yellow crystals; m.p. $305^{\circ} \mathrm{C}$; IR: $v=3500-3400$ (carbonyl overtone, $\mathrm{C}=\mathrm{O}$ ), 3040 (aromatic $\mathrm{C}-\mathrm{H}$ ), 2935 (aliphatic $\mathrm{C}-\mathrm{H}$ ), 1745-1660-1650 (C=O), 1600-1450 (aromatic ring, skeleton vib.), 1245 (C=S), 1220-1050 (anhydride C-O stretch), 800700 (pyrimidine ring); ${ }^{1} \mathrm{H}$ NMR (DMSO- $\left.d_{6}\right)$ : $\delta=9.01 \mathrm{ppm}(\mathrm{s}$,
$1 \mathrm{H}$, pyrimidine ring), 7.90-6.79 ( $\mathrm{m}, 15 \mathrm{H}$, aromatic), 2.35-2.10 ppm (s, 6H, 2x-CH $)_{3}$ ) ${ }^{13} \mathrm{C}$ NMR (DMSO- $\left.d_{6}\right): \delta=187.51$ (s, benzoyl's $\mathrm{C}=\mathrm{O}$ ), 170.50 (s, pyrimidine ring, $\mathrm{C}=\mathrm{S}$ ), 170.10 (s, anhydride's $2(\mathrm{C}=\mathrm{O}), 144.01-118.68(\mathrm{~m}$, aromatic $\mathrm{C}), 23.15$, $21.45 \mathrm{ppm}\left(\mathrm{s}, 2 \times \mathrm{CH}_{3} \mathrm{C}_{6} \mathrm{H}_{4}-\right)$. Elemental analysis (\%): Found (calcd.): $\mathrm{C}=72.32$ (72.15), $\mathrm{H}=4.01$ (4.26), $\mathrm{N}=9.18$ (9.35).

$\mathrm{N}$-[5-(4-Methylbenzoyl)-4-(4-methylphenyl)-2oxopyrimidin-1(2H)-yl]benzamide (3e): $0.2 \mathrm{~g}$ of 1-Amino5-(4-methylbenzoyl)-4-(4-methylphenyl)pyrimidine-2(1H)one (1a) and $1.4 \mathrm{~g}$ benzoic anhydride (2c) (1: 10 molar ratio) were homogeneously mixed. The mixture was heated at $80^{\circ} \mathrm{C}$ for at $2 \mathrm{~h}$ without any solvent. After cooling to room temperature the residue was treated with dry ether and the formed crude product recrystallized from n-butanol; yield: $0.18 \mathrm{~g}$ $(68 \%)$, white crystals; m.p. $270{ }^{\circ} \mathrm{C}$; IR: $v=3500-3400$ (carbonyl overtone, $3203(-\mathrm{N}-\mathrm{H}), \mathrm{C}=\mathrm{O}), 3040$ (aromatic C-H), 2945 (aliphatic C-H), 1750-1660-1645 (C=O), 15801520 (aromatic ring, skeleton vib.), $1235(\mathrm{C}=\mathrm{S}), 1220-1050$ (anhydride C-O stretch), 800-700 (pyrimidine ring); ${ }^{1} \mathrm{H}$ NMR (DMSO- $\left.d_{6}\right): 12.11(\mathrm{~s}, 1 \mathrm{H}, \mathrm{N}-\mathrm{H}), \delta=8.96 \mathrm{ppm}(\mathrm{s}, 1 \mathrm{H}$, pyrimidine ring), 7.95-6.70 (m, 13H, aromatic), 2.35-2.20 ppm (s, 6H, $\left.2 \mathrm{x}-\mathrm{CH}_{3}\right) ;{ }^{13} \mathrm{C} \mathrm{NMR}\left(\mathrm{DMSO}-d_{6}\right): \delta=190.51$ (s, benzoyl's $\mathrm{C}=\mathrm{O}$ ), 167.10 (s, amide, $\mathrm{C}=\mathrm{O}$ ), 151.30 (s, pyrimidine ring, $\mathrm{C}=\mathrm{O}$ ), 138.39-114.90 (m, aromatic $\mathrm{C}), 23.15,21.14 \mathrm{ppm}(\mathrm{s}$, $\left.2 \mathrm{xCH}_{3} \mathrm{C}_{6} \mathrm{H}_{4}-\right)$. Elemental analysis (\%): Found (calcd.): $\mathrm{C}=$ 73.55 (73.74), $\mathrm{H}=4.83$ (5.00), $\mathrm{N}=9.79$ (9.92).

$\mathrm{N}$-[5-(4-Methylbenzoyl)-4-(4-methylphenyl)-2-thioxopyrimidin-1(2H)-yl]benzamide (3f): $0.2 \mathrm{~g}$ of 1-Amino-5-(4methylbenzoyl)-4-(4-methylphenyl)pyrimidine-2(1H)-thione (1b) and $1.34 \mathrm{~g}$ benzoic anhydride (2c) (1: 10 molar ratio) were homogeneously mixed. The mixture was heated at $80^{\circ} \mathrm{C}$ for at $3 \mathrm{~h}$ without any solvent. After cooling to $0.16 \mathrm{~g}$ (64\%), yellow crystals; m.p. $270{ }^{\circ} \mathrm{C}$; IR: $v=3500-3400$ (carbonyl overtone, $3220(-\mathrm{N}-\mathrm{H}), \mathrm{C}=\mathrm{O}), 3050$ (aromatic C-H), 2940 (aliphatic C-H), 1750-1660 (C=O), 1600-1540 (aromatic ring, skeleton vib.), 1220-1050 (anhydride C-O stretch), 800-700 (pyrimidine ring); ${ }^{1} \mathrm{H}$ NMR (DMSO- $\left.d_{6}\right): 12.11(\mathrm{~s}, 1 \mathrm{H}, \mathrm{N}-\mathrm{H})$, $\delta=8.96 \mathrm{ppm}(\mathrm{s}, 1 \mathrm{H}$, pyrimidine ring), 7.95-6.70 (m, $13 \mathrm{H}$, aromatic), 2.35-2.20 ppm (s, 6H, 2x-CH $)$; ${ }^{13} \mathrm{C} \mathrm{NMR} \mathrm{(DMSO-}$ $\left.d_{6}\right): \delta=190.51$ (s, benzoyl's $\left.\mathrm{C}=\mathrm{O}\right), 172.30$ (s, pyrimidine ring, $\mathrm{C}=\mathrm{S}$ ), 167.10 (s, amide, $\mathrm{C}=\mathrm{O}$ ), 140.39-110.10 (m, aromatic C), 22.40, $20.10 \mathrm{ppm}\left(\mathrm{s}, 2 \mathrm{xCH}_{3} \mathrm{C}_{6} \mathrm{H}_{4}-\right)$. Elemental analysis (\%): Found (Calcd.): $\mathrm{C}=71.25$ (71.05), $\mathrm{H}=4.70$ (4.82), $\mathrm{N}=9.32$ (9.56), $\mathrm{S}=7.18$ (7.30).

1-[5-(4-Methylbenzoyl)-4-(4-methylphenyl)-2oxopyrimidin-1 $(2 H)$-yl]-1 $H$-pyrrole-2,5-dione (3g): $0.2 \mathrm{~g}$ of 1-Amino-5-(4-methylbenzoyl)-4-(4-methylphenyl)pyrimidine2(1H)-one (1a) and $0.61 \mathrm{~g}$ maleic anhydride (2d) (1: 10 molar ratio) were heated at $50{ }^{\circ} \mathrm{C}$ for at $2 \mathrm{~h}$ without any solvent. Then, the residue was treated with dry ether and filtered and the so formed crude product was recrystallized from $n$ butanol; yield $0.15 \mathrm{~g}(65 \%)$, yellow crystals; m.p. $330{ }^{\circ} \mathrm{C}$ IR: $v=3045$ (aromatic C-H), 2950 (aliphatic C-H), 1730-1655$1640(\mathrm{C}=\mathrm{O}), 1600-1520(\mathrm{C}=\mathrm{C}$ and $\mathrm{C}=\mathrm{N}), 1220-1050$ (anhydride's C-O stretch), 800-700 $\mathrm{cm}^{-1}$ (pyrimidine ring); ${ }^{1} \mathrm{H}$ NMR (DMSO- $\left.d_{6}\right): \delta=9.01$ (s, $1 \mathrm{H}$, pyrimidine ring.), 7.806.53 (m, 10H, ArH), 2.35, 2.10 ppm (s, 6H, 2x-CH3); ${ }^{13} \mathrm{C} \mathrm{NMR}$ (DMSO- $d_{6}$ ): $\delta=190.50$ (s, benzoyl group, $\mathrm{C}=\mathrm{O}$ ), 160.30 (s, 
anhydride's $2(\mathrm{C}=\mathrm{O}), 151.30$ (s, pyrimidine ring, $\mathrm{C}=\mathrm{O}), 141.01$ 118.20 (m, aromatic C), 130.15 (d, $-\mathrm{CH}=\mathrm{CH}-), 21.80,21.20$ ppm (s, $\left.2 \mathrm{xCH}_{3} \mathrm{C}_{6} \mathrm{H}_{4}-\right)$. Elemental analysis (\%): Found (calcd.): $\mathrm{C}=67.81$ (67.92), $\mathrm{H}=3.42$ (3.53), $\mathrm{N}=11.10$ (11.32).

1-[5-(4-Methylbenzoyl)-4-(4-methylphenyl)-2-oxopyrimidin-1(2H)-yl]-1H-pyrrole-2,5-dione (3h): $0.2 \mathrm{~g}$ of $0.2 \mathrm{~g}$ 1-Amino-5-(4-methylbenzoyl)-4-(4-methylphenyl)pyrimidine-2(1H)-thione (1b) and $0.58 \mathrm{~g}$ maleic anhydride (2d) (1: 10 molar ratio) were heated at $50{ }^{\circ} \mathrm{C}$ for at $3 \mathrm{~h}$ without any solvent. Then, the residue was treated with dry ether and filtered and the so formed crude product was recrystallized from n-butanol; yield $0.14 \mathrm{~g}(61 \%)$, yellow crystals; m.p. $330{ }^{\circ} \mathrm{C}$; IR: $v=3035$ (aromatic C-H), 2940 (aliphatic C-H), 1745-1660 $(\mathrm{C}=\mathrm{O}), 1595-1490(\mathrm{C}=\mathrm{C}$ and $\mathrm{C}=\mathrm{N}), 1235(\mathrm{C}=\mathrm{S})$, 1220-1050 (anhydride's C-O stretch), 800-700 $\mathrm{cm}^{-1}$ (pyrimidine ring.); ${ }^{1} \mathrm{H}$ NMR (DMSO- $\left.d_{6}\right): \delta=8.91(\mathrm{~s}, 1 \mathrm{H}$, pyrimidine ring), 7.90-6.83 (m, 10H, ArH), 2.35, 2.10 ppm (s, 6H, $\left.2 \mathrm{x}-\mathrm{CH}_{3}\right) ;{ }^{13} \mathrm{C}$ NMR (DMSO- $\left.d_{6}\right): \delta=190.20$ (s, benzoyl C=O), 169.30 (s, pyrimidine ring, $\mathrm{C}=\mathrm{S}), 160.30$ (s, anhydride's $2(\mathrm{C}=\mathrm{O}), 142.01$ 110.20 (aromatic C), 131.20 (-CH=CH-), 23.20, 21.40 ppm (s, $\left.2 \mathrm{xCH}_{3} \mathrm{C}_{6} \mathrm{H}_{4}-\right)$. Elemental analysis (\%): Found (calcd.): $\mathrm{C}=$ 62.05 (62,21), $\mathrm{H}=3.78$ (3.72), $\mathrm{N}=10.36$ (10.16), $\mathrm{S}=7.90$ (7.60).

\section{RESULTS AND DISCUSSION}

The reactions of 1-amino-5-(4-methylbenzoyl)-4-(4methylphenyl)pyrimidine-2(1H)-one/-thione (1a,b) with acid anhydride derivatives (2a-e) yielded $N$-acyl derivatives ${ }^{11}$ (3a-h). Several amides and imide derivatives (3a-h) were easily obtained in moderate yields (57-68 \%) from nucleophilic addition of $\mathbf{1 a}, \mathbf{b}$ to the corresponding compounds of $\mathbf{2 a - d}$ (Scheme-I). The reactions were performed by heating them without any solvent up to $50-190^{\circ} \mathrm{C}$ (see experimental section). The structure of $\mathrm{N}$-[5-(4-methylbenzoyl)-4-(4-methylphenyl)2-oxopyrimidine-1(2H)-yl]pyrrolidine-2,5-dione (3a) was confirmed by its elemental analysis, IR, ${ }^{1} \mathrm{H}$ NMR and ${ }^{13} \mathrm{C}$ NMR specroscopic data (Scheme-I). The absorption bands of $(\mathrm{C}=\mathrm{O})$ groups were found to be at 1720, 1700, 1670, $1650 \mathrm{~cm}^{-1}$. The absorption band of aliphatic $\mathrm{C}$-H groups were found to be at $2930 \mathrm{~cm}^{-1}$. The ${ }^{1} \mathrm{H}$ NMR signals were found to be at $\delta=8.90$ (s, $1 \mathrm{H}$, pyrimidine ring) and 7.77-6.84 ppm (m, 8H, ArH). The ${ }^{1} \mathrm{H}$ NMR spectrum showed peaks of $-\mathrm{CH}_{2}-\mathrm{CH}_{2-}$ at $\delta=$ 2.60-2.45 and 2.25, 2.05 ppm (s, $\left.6 \mathrm{H}, 2 \mathrm{x}-\mathrm{CH}_{3}\right)$. The ${ }^{13} \mathrm{C}$ NMR signals were observed at $\delta=191.50$ (benzoyl group, $\mathrm{C}=\mathrm{O}$ ), $162.30 \mathrm{ppm}$ (anhydride's $2(\mathrm{C}=\mathrm{O})$. Finally, the elemental analysis data along with spectroscopic data (details see experimental part) confirm the structure of 3a. The results of measurements of $\mathbf{3 b} \mathbf{b}$ were given in experimental section.

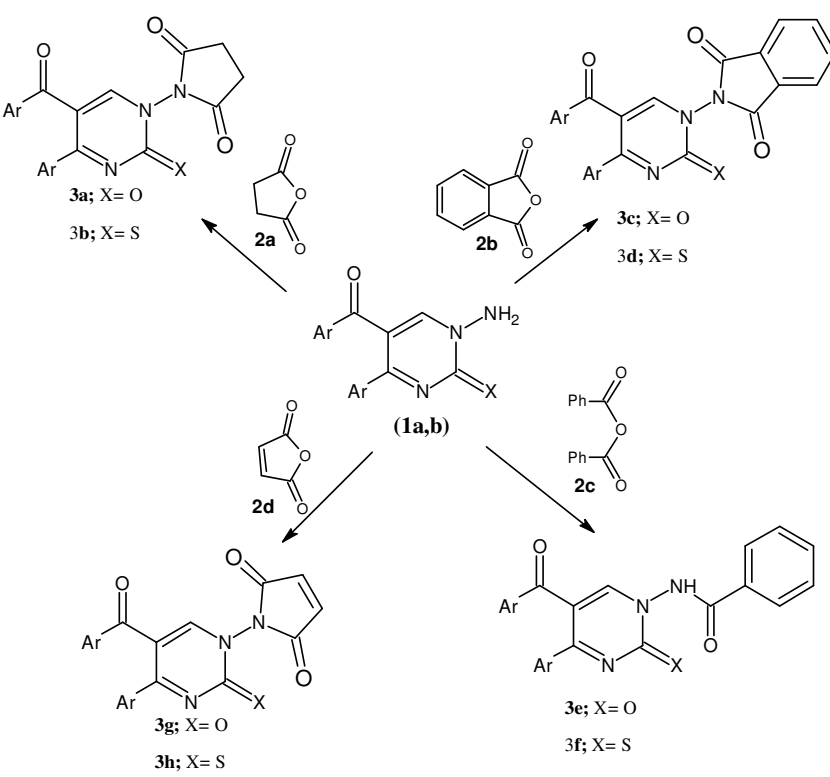

Scheme-I

\section{ACKNOWLEDGEMENTS}

The author is grateful for the financial support given by the Research Foundation of Erciyes University.

\section{REFERENCES}

1. R.S. Varma, Green Chem., 1, 43 (1999).

2. C.O. Kappe, Tetrahedron, 46, 6937 (1993).

3. W. Xie, Y. Jin and P.G. Wang, Chemtech., 2, 23 (1999).

4. C.O. Kappe, W.M.F. Fabian and M.A. Semones, Tetrahedron, 53, 2803 (1997).

5. G.J. Grover, S. Dzwonczyk, D.M. Mc Mullen, C.S. Normadinam, P.G. Sleph and S.J. Moreland, Cardiovasc. Pharmacol., 26, 289 (1995).

6. C.D. Selassie, R. Poe, M. Li and C. Hansch, J. Med. Chem., 34, 46 (1991).

7. E.L. Burdge, Pest Manag. Sci., 56, 245 (2000).

8. K. Parfitt, Martindale, Pharmaceutical Press, London, edn 32 (1999).

9. I. Yildirim and I. Koca, Kuwait J. Sci. Eng., 32, 49 (2005).

10. Z. Önal and I. Yildirim, Heterocycl. Commun., 13, 113 (2007).

11. Z. Önal, E. Saripinar and I.Ö. Ilhan, J. Heterocycl. Chem., 38, 397 (2001).

12. Z. Önal and B. Altural, Turk. J. Chem., 23, 401 (1999).

13. Z. Önal and A.C. Daylan, Asian J. Chem., 19, 1455 (2007).

14. Z. Onal, N. Atli and I.O. Ilhan, Heterocycl. Commun., 15, 385 (2009).

15. Z. Onal, H. Ceran and E. Sahin, Heterocycl. Commun., 14, 245 (2008).

16. Z. Onal, I. Yildirim, F. Kandemirli and T. Arslan, Struct. Chem., 21, 809 (2010).

17. Z. Onal, H. Ceran and E. Sahin, Heterocycl. Commun., 14, 245 (2008). 